\title{
Direct Observation of Capacitor Switching Using Planar Electrodes
}

\author{
By Nina Balke,* Martin Gajek, Alexander K. Tagantsev, Lane W. Martin, Ying-Hao Chu, \\ Ramamoorthy Ramesh, and Sergei V. Kalinin
}

Ferroelectric polarization switching in epitaxial (110) $\mathrm{BiFeO}_{3}$ films is studied using piezoresponse force microscopy of a model in-plane capacitor structure. The electrode orientation is chosen such that only two active domain variants exist. Studies of the kinetics of domain evolution allows clear visualization of nucleation sites, as well as forward and lateral growth stages of domain formation. It is found that the location of the reverse-domain nucleation is correlated with the direction of switching in a way that the polarization in the domains nucleated at an electrode is always directed away from it. The role of interface charge injection and surface screening charge on switching mechanisms is explored, and the nucleation is shown to be controllable by the bias history of the sample. Finally, the manipulation of domain nucleation through domain structure engineering is illustrated. These studies pave the way for the engineering and design of the ferroelectric device structures through control of individual steps of the switching process.

\section{Introduction}

Ferroelectrics are rapidly emerging as an enabling component of non-volatile information technology devices, including ferroelectric random access memory (FeRAM), , ${ }^{[1]}$ field-effect transistors, ${ }^{[2-4]}$ and tunneling barriers. ${ }^{[5-8]}$ In most applications,

[*] Dr. N. Balke, Dr. S. V. Kalinin

Center for Nanophase Materials Sciences

Oak Ridge National Laboratory

Oak Ridge, TN, 37831, USA

E-mail: balken@ornl.gov

Dr. M. Gajek, Prof. R. Ramesh

Department of Physics

University of California

Berkeley, Berkeley, CA, 94720, USA

Prof. A. K. Tagantsev

Ceramics Laboratory

Ecole Polytechnique Federale de Lausanne

1015 Lausanne, Switzerland

Prof. L. W. Martin

Department of Materials Science and Engineering

University of Illinois

Urbana-Champaign, Urbana, IL 61801, USA

Prof. Y.-H. Chu

Department of Materials Science and Engineering

National Chiao Tung University

Hsinchu 30010, Taiwan the functionality is based on capacitor- or tunneling barrier type structures formed by a ferroelectric embedded between two metallic electrodes. The lateral size of the structure determines the ultimate storage density of the device, thus necessitating the continuous drive for device miniaturization. The use of e-beam writing has allowed structures down to a $100 \mathrm{~nm} \times 100 \mathrm{~nm}$ cell size to be fabricated, ${ }^{[9]}$ with even smaller structures being developed using templated growth ${ }^{[10]}$ and self-assembly processes. Correspondingly, understanding the switching behavior in capacitor structures and the role of microstructure and interfaces on this process is crucial in determining the application limits and in predicting long-term operation.

Typical capacitor investigations include measuring switching currents or polarization $P$ as function of switching field and time. ${ }^{[11-16]}$ These yield spatially-averaged information on rate limiting steps and switching mechanisms; however, no direct information on the spatial uniformity of switching behavior and the role of defects and lateral confinement on switching can be obtained. Recently, piezoresponse force microscopy (PFM) has emerged as a key tool to characterize ferroelectric capacitors ${ }^{[17-22]}$ by providing the capability to visualize domain patterns at different stages of the switching process. Recent developments in the field of capacitor investigations using PFM include time resolved measurement using very short switching pulses, ${ }^{[23]}$ and switchingspectroscopy PFM (SSPFM) to investigate the capacitor switching at different positions on the capacitor. ${ }^{[24]}$ However, the vast majority of PFM studies have addressed capacitors in the stack geometry, with the imaging plane coinciding with the top electrode (more specifically, top electrode-ferroelectric interface). In this case, the information on the depth distribution of polarization is essentially lost. This issue was partially overcome by Hong et al. who used chemical etching to remove layers of material to access different capacitor depths ${ }^{[25]}$ and the use of beveled ferroelectric structures by Stolichnov ${ }^{[26]}$ and $\mathrm{Lu}$ et al. ${ }^{[27]}$ However, the influence of the etching, as well as deviations from uniform field distribution, on the domain structure and switching process is not clear and the characterization process is destructive. An alternative approach to investigate capacitor switching is based on using planar or in-plane electrodes to generate electric fields parallel to the sample surface, with the PFM probe used to observe the evolution of domain 
structures. ${ }^{[28,29]}$ The advantage of planar electrode configuration is that the switching process between the electrodes can be observed directly and that the piezoelectric response is not averaged over the film thickness.

In this communication, we report the detailed study of capacitor switching mechanisms using multiferroic $\mathrm{BiFeO}_{3}$ (BFO) epitaxially grown on $\mathrm{SrTiO}_{3}$ as a model system. A planar electrode configuration was used to enable observations of the full switching process with a PFM tip, without losing any depth information. We demonstrate the role of domain nucleation and domain wall motion in the capacitor switching process and investigate the role of electrical charges on ferroelectric switching. This represents the first time the switching process has been observed between two electrodes, enabling direct observation and identification of the switching processes.

\section{Switching of the Pristine Capacitors: Nucleation and Wall Pinning}

\subsection{Domain Structures and In-Plane Switching}

For the planar capacitor samples, buried $50 \mathrm{~nm}$ thin $\mathrm{SrRuO}_{3}$ electrodes underneath $80 \mathrm{~nm}$ thin (110)-oriented BFO films were used. A schematic of the capacitor structure along with the topography line scan to show the quality of the etched capacitor edges are shown in Figure 1a and Figure 1b. Comparable sample structures were also used by Shafer et al. ${ }^{[30]}$ and Chu et al. ${ }^{[31]}$ for (001)-oriented BFO. Structures studied in this report were designed to have a $6 \mu \mathrm{m}$ electrode spacing (note that electrode separation yields a length scale marker for the Figures throughout the paper). The buried electrodes allow for the application of the relatively high fields (in excess of $6 \mathrm{kV} \mathrm{cm}^{-1}$ ) necessary to switch the capacitor, while avoiding arching and associated ferroelectric-metal interface damage. In addition, the use of the buried electrode structure precludes the damage to the ferroelectric film due to etching or material lift-off during device fabrication (which is known to strongly change the surface state of the material). The BFO films were considerably thinner than the electrode spacing to ensure the formation of through-thickness domains.

The typical equilibrium as-grown state of a (110)-oriented BFO films is characterized by two possible upward-oriented polarization variants separated by $71^{\circ}$ domain walls. ${ }^{[32,33]}$ The cantilever is oriented along the [110] axis to distinguish the two in the in-plane (IP) PFM image. The out-of-plane (OP) PFM was used to confirm the absence of out-of plane switching. The edges of the in-plane electrodes are also oriented parallel to the $[1 \overline{1} 0]$ direction so that the applied in-plane field switches the BFO between the two naturally occurring in-plane domain variants. Figure $1 \mathrm{c}$ and Figure $1 \mathrm{~d}$ show the topography, the as-grown OP and IP PFM images and a schematic unit cell showing the two domain variants. Note that the as-grown domain pattern is different on top of the $\mathrm{SrRuO}_{3}$ electrodes and on the $\mathrm{SrTiO}_{3}$ due to the difference in bottom electrode boundary condition.

Prior to switching studies, the capacitors were poled using $\pm 100 \mathrm{~V}$ for several seconds and the resulting domain patterns are shown in Figure 2a. At these biases, the capacitor can be switched almost completely, with a small number of residual domains at the positive side of the poling field. The OP PFM images (not shown) do not exhibit any domain contrast, thus

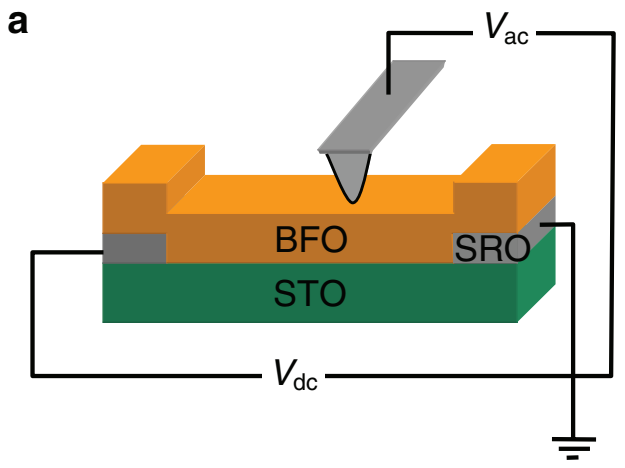

C

Topograph
OP PFM

\section{$\circ$}

b
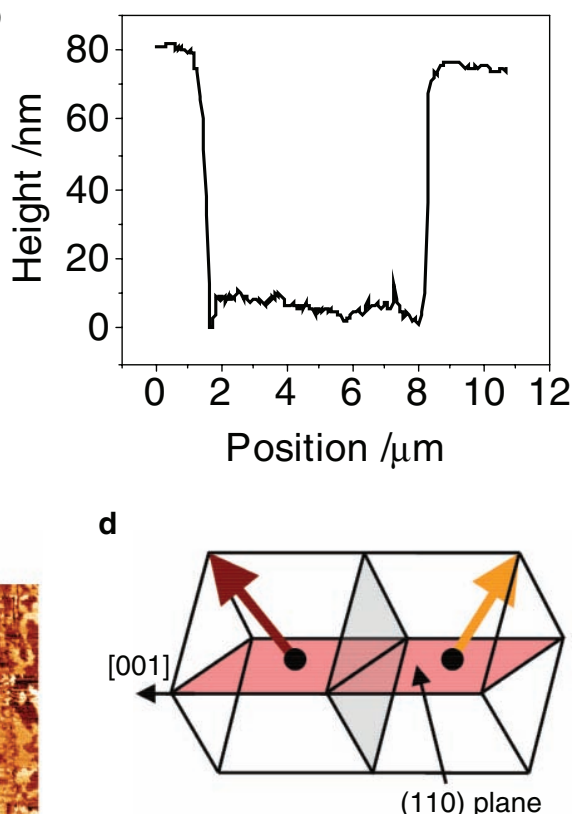

Figure 1. a) Schematic of the sample structure with indication where poling fields (Vdc) and imaging fields (Vac) are applied. b) Linescan over sample structure. c) Topography, OP PFM and IP PFM images of the as-grown sample with indication of cantilever and domain orientation. d) Schematic of unit cell. The scale bar for all PFM images can be extracted from the fact that the electrode gap of the capacitor is always $6 \mu \mathrm{m}$. 
a

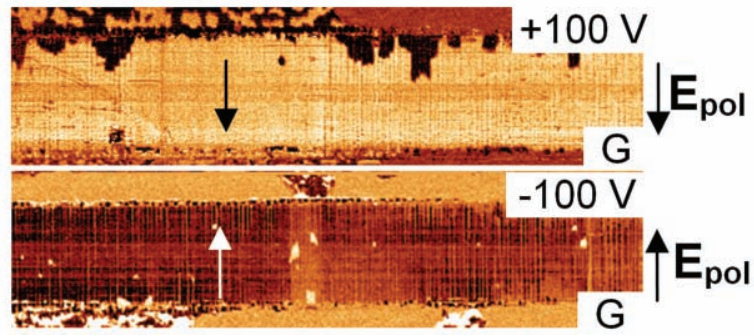

b

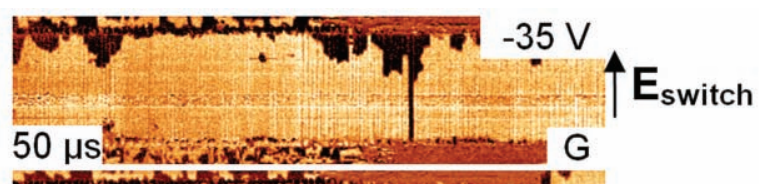

C

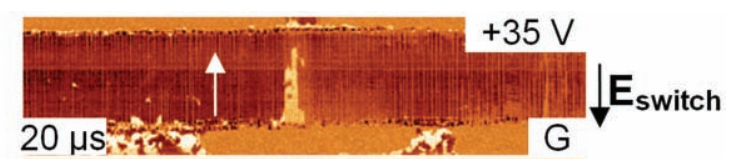

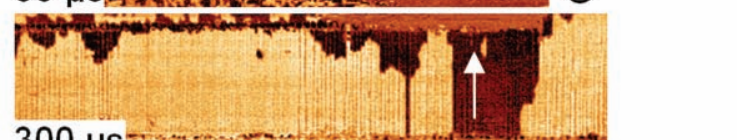
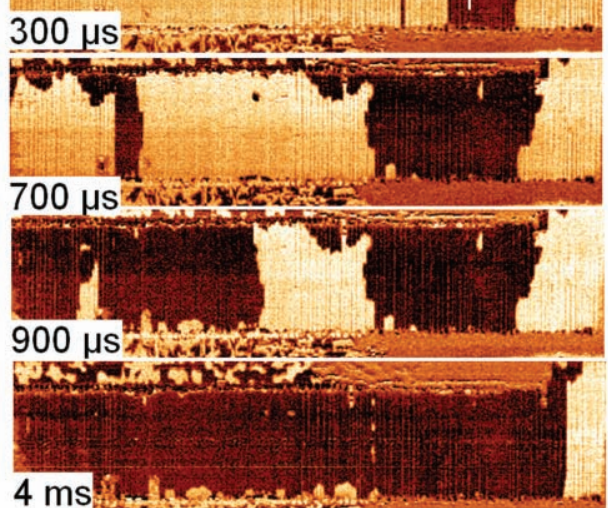

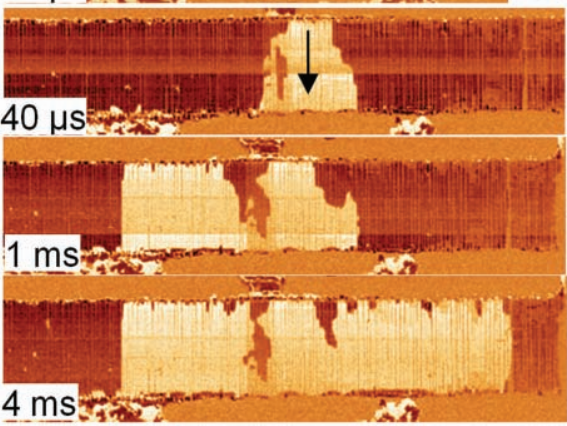

d

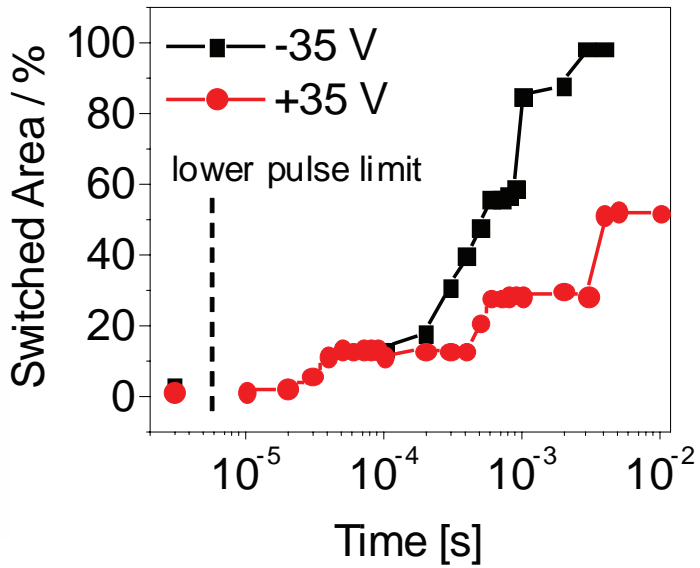

Figure 2. a) IP PFM images of the same capacitor after poling with $+100 \mathrm{~V}$ or $-100 \mathrm{~V}$. IP PFM images showing the switching sequence for b) negative and c) positive switching voltages. d) Switched domain area as function of switching pulse lengths for positive and negative voltages showing the asymmetric capacitor switching.

indicating that the switching is purely in-plane and only two domain orientations are involved in a $71^{\circ}$ switching process. Due to this small number of active domain variants and welldefined switching process, this system offers an ideal model system for high-resolution cross-sectional studies of capacitor switching.

\subsection{Time-Resolved Switching Imaging}

The domain evolution during the switching sequence with application of $-35 \mathrm{~V}$ pulses at varying pulse lengths (no repoling between the pulses) is shown in Figure $2 \mathrm{~b}$. The ferroelectric domain structure was imaged after each voltage pulse, clearly illustrating the classical capacitor switching mechanism. In the first stage, the domains nucleate and rapidly grow forward until they reach the opposite electrode. Second, the domain walls grow by sideway growth until the whole capacitor area is switched. In total, there are three domain nucleation sites over the $35 \mu \mathrm{m}$ length of the electrodeferroelectric interface imaged here. The onset of sideways domain growth is different for all three nucleation sites (see for example $300 \mu \mathrm{s}$ ) and the growth occurs discontinuously, suggesting the presence of multiple pinning sites in the capacitor.

The sequence of events during the switching of the capacitor in the opposite direction with positive voltages $(35 \mathrm{~V})$ is shown in Figure 2c. Comparing Figure $2 \mathrm{~b}$ and $\mathrm{c}$ illustrates that the domain nucleation initiating the polarization reversal in the capacitor happens at the electrode the switching field is pointing at. This means, only domain walls with a nominal negative charge form (see also Figure 3a,b). The switched area grows with increasing duration of the switching pulse (Figure 2d), however, for the switching voltage of $+35 \mathrm{~V}$ switching is typically incomplete. Nearly full switching can be achieved as shown in Figure 2a "-100 V" with higher poling voltages.

Notably, the remnants of non-reversed polarization left after poling with a voltage of given sign do not move when the switching voltage of the opposite sign is applied. Thus, these residual domains do not act as "seeds" for the polarization reversal, contrary to expectations (Figure $2 \mathrm{~b}$ and Figure $3 \mathrm{c}$ ). Furthermore, the sideways domain growth can actually be hindered by the residual domains, which thus act as pinning centers as shown in Figure 3c. Repeated switching of the capacitors 
a

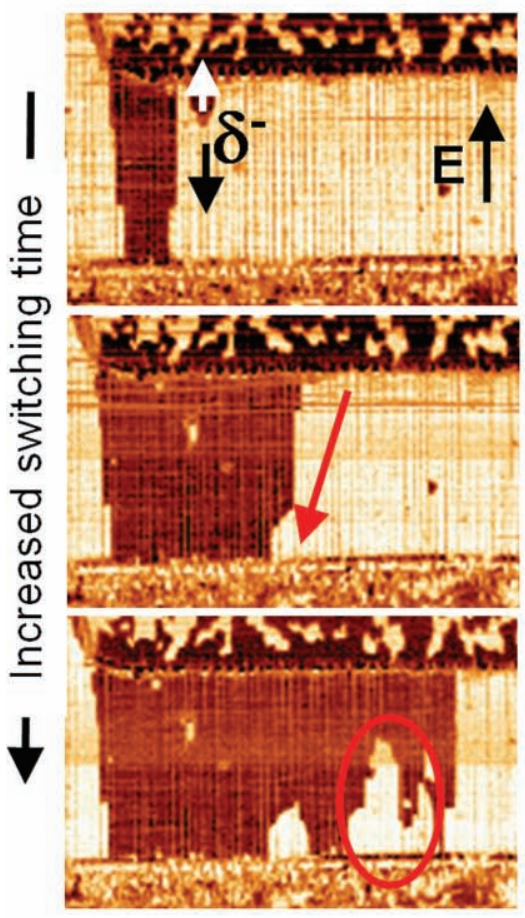

b
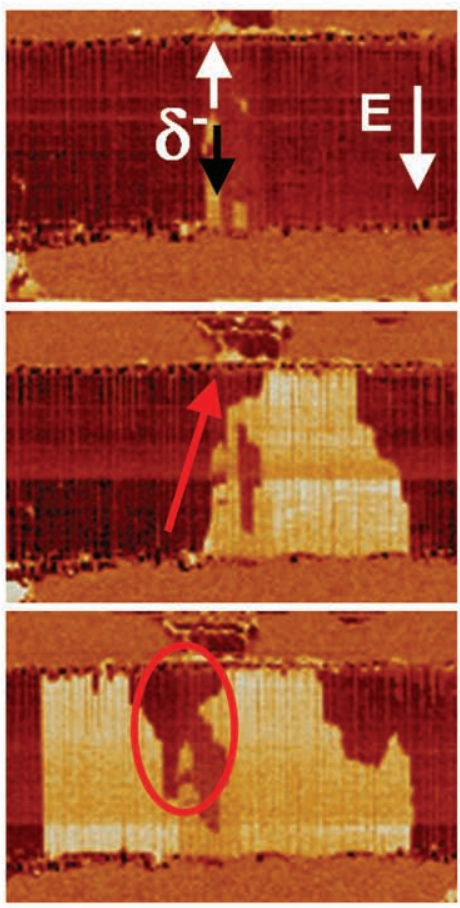

C
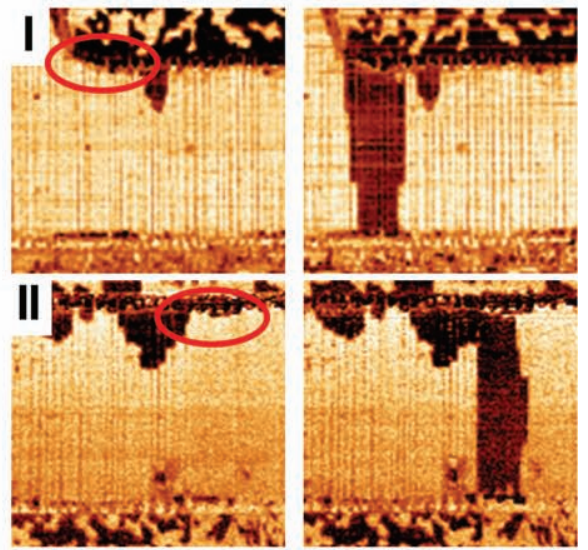

III

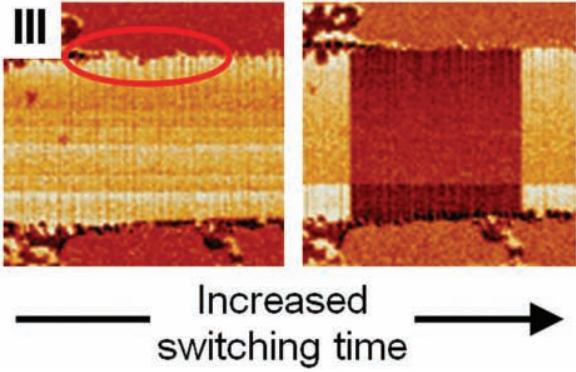

Figure 3. Forming of negatively charged domain walls for switching with a) negative and b) positive voltages. With increasing switching time the domains become narrower towards the positively charged electrode (red arrows) and residual domains are only formed on the positive electrode (red circles) suggesting that switching starts from the negatively charged electrode. c) Three examples to show that domain nucleation sites differ from the location of residual domains (red circles) and that sideways domain wall motion is stopped at residual domains.

showed that the nucleation or pinning sites are not always exactly the same but appear approximately in the same regions.

The third important aspect of the switching process is the role of the surface morphology of the film. The topography of (110)-oriented BFO shows stripes along the [001] direction that are the result of surface energy minimization during film growth on this orientation. ${ }^{[34]}$ In Figure $2 b$ it was shown that very thin domains grow from one electrode to the other before sideways domain wall growth takes place. These domains always have the same width and appear to be determined by the spacing of the topographical features. This is shown in Figure 4, where topography, IP phase, and amplitude PFM images are shown together with line scans over two topographical stripes. It can be seen that the domain walls follow exactly the topography minima and that the domain width is a multiple of the minima spacing.

\section{Manipulation of Capacitor Switching}

\subsection{Electrical Cycling and Imprint}

Beyond the role of topography in the nucleation mechanism, we explored the role of ac field training (cycling) and imprint (i.e. the effect of long-term dc poling on the material switching). The impact of ac cycling on the switching kinetics has been investigated by cycling the capacitor with $\pm 50 \mathrm{~V}$ at $0.5 \mathrm{~Hz}$. The switching behavior of the capacitor was characterized after 10 and 100 cycles. After 10 cycles, the overall switching behavior (switched area vs. time) is comparable with the first switching cycle. After 100 cycles, however, the switching behavior was strongly altered (Figure 5). The electrical cycling reduces the nucleation voltage and increases the domain wall velocity for the lateral domain wall motion. For example, during the first switching with $-35 \mathrm{~V}$ a pulse length of $200 \mu$ s was necessary to induce significant switching. After 100 electrical cycles a comparable degree of switching was already achieved with a pulse length of $10 \mu \mathrm{s}$. The number of nucleation sites is comparable in both cases (not shown here), which demonstrate that the ac field training only affected the nucleation bias and wall velocity for sideways growth. The analysis of the switched domain area as function of switching pulse length for the first and 100th switching cycle is shown in Figure 5a, illustrating that the switching curve is shifted by an order of magnitude towards faster switching times.

This training effect also changes the capacitor switching with positive voltages as shown in Figure $5 \mathrm{~b}$. During the first switching cycle $35 \mathrm{~V}$ was not high enough to switch the capacitor completely (Figure 2c). After 100 cycles, the capacitor switches completely with the same switching voltage and the switching times stay the same as before. However, the effect of cycling is only temporarily and after two days relaxation the switching characteristics are approximately the same as for the first switching cycle, suggesting that this effect originates 
a

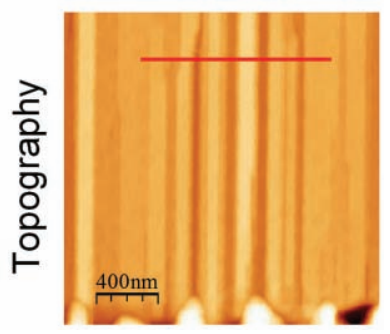

b

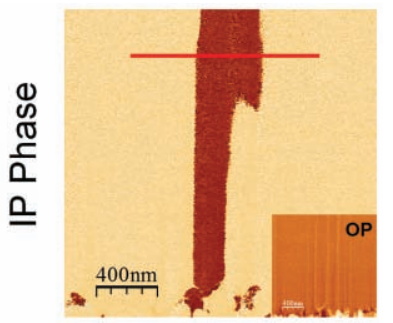

C

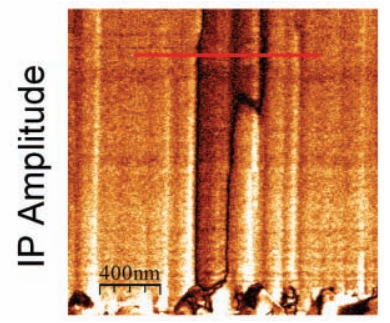

d

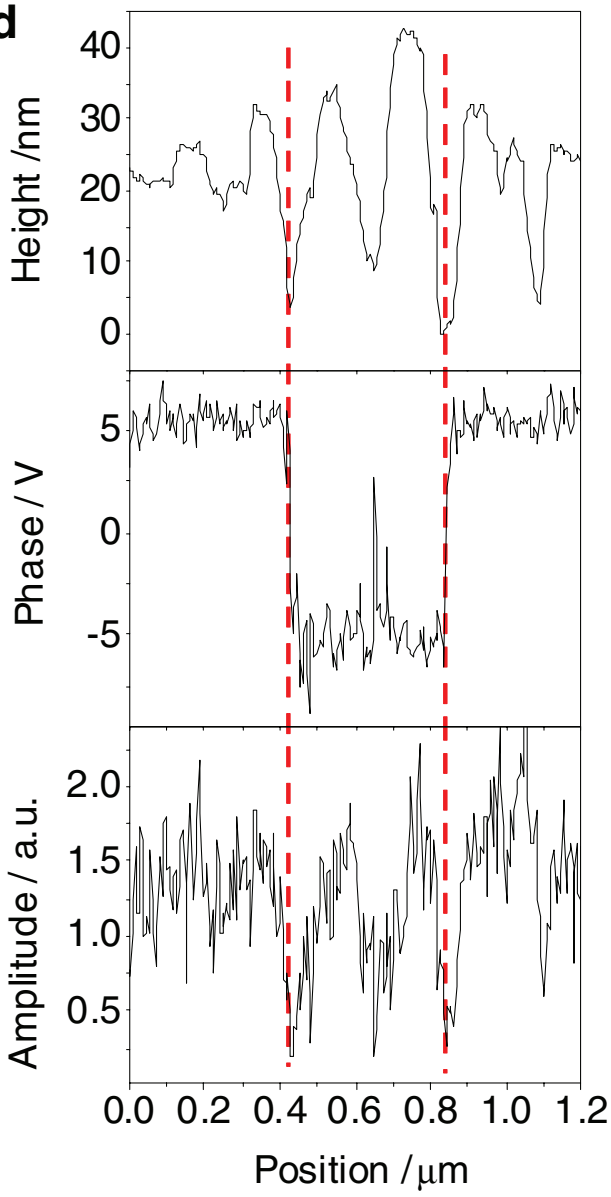

Figure 4. a) Topography, b) IP phase, and c) IP amplitude for a domain after forward growth and the d) corresponding linescans showing the correlation between domain walls and topography.

from the injection of relatively short-lived charged at the electrode-ferroelectric interface. The training effect discussed above is comparable to the one observed in ferroelectric ceramics and thin films. ${ }^{35-37]}$

Finally, the imprint effect has been addressed comparing the switching kinetics of a capacitor that was poled using a short positive voltage pulse (as in Figure 2a) and switched with $-35 \mathrm{~V}$ and a capacitor that was pre-poled (or imprinted) with $-50 \mathrm{~V}$ for $10 \mathrm{~min}$ prior to the standard switching procedure. The data shown in Figure 6 display the influence of the imprinting on the capacitor switching characteristics. After the same switching conditions of $-35 \mathrm{~V}$ and $10 \mu \mathrm{s}$ the number of nucleation sites is strongly increased for the imprinted capacitor (Figure 6b) if the imprint field was applied in the same direction as the switching field. However, the sideways domain wall motion is less affected by the imprint as can be seen in Figure $6 \mathrm{c}$ where the switched capacitor area as function of switching time is shown. The switching curve for the imprinted capacitor is slightly shifted along the area axis in comparison to the non-imprinted capacitor. Such imprint behavior is comparable to that reported to for $\mathrm{BaTiO}_{3}{ }^{[38]}$ and $\mathrm{Pb}\left(\mathrm{Zr}_{x}, \mathrm{Ti}_{1-x}\right) \mathrm{O}_{3}{ }^{[39-41]}$ parallel plate thin film capacitors.

\subsection{Role of Biasing Conditions}

In the following, we explore whether the stability of negatively charged domain walls can be modified by the choice of the biasing conditions. For capacitor switching, the field direction of the switching field and the voltage sign used to establish the field can be controlled independently, allowing the interplay between switching process and surface charging to be explored. Figure 7a shows two configurations that can be used to create the same field. In the 'normal' setup, a negative voltage is applied to one of the electrodes in which case the grounded electrode is positively charged relative to the negative voltage. The switching field points towards the negatively charged electrode. The same field direction can be applied if ground is connected to the previous negatively charged electrode and a positive voltage is applied to the opposite electrode. In this "reversed" configuration, the ground side acts as the negative electrode. Depending on the combination of poling and switching setup, the IP PFM images after switching with $|35 \mathrm{~V}|$ and $10 \mu$ s are quite different, which is shown in Figure 8b-e. In the case of switching with the normal setup and if both poling and switching is done with the reversed setup, the images are identical (Figure 8b,c,e). However, if the poling was done with the normal setup but switching afterwards with the reversed setup, the degree of switching under the same conditions is highly increased. The analysis of the domain orientation shows, that in this case the domain walls are positively charged. The measurement has been done several times and is reproducible. If the switching is done after a waiting time of two minutes after poling, the effects of increased switching area and the formation of positively charged domain walls are gone.

\subsection{Local Manipulation of the Domain Structure}

As shown in chapter 2.2, residual domains do not take part in the capacitor switching process. In order to explain this behavior, artificial residual domains were switched locally using a biased scanning probe. The probe was scanning parallel, next to one of the electrodes, and the bias was applied between tip and the electrode next to the tip. The result is shown in Figure 8a, where a residual domain was switched parallel to the top electrode across its whole length. After switching with $-35 \mathrm{~V}$ for $5 \mathrm{~ms}$, no capacitor switching takes place (compare with Figure $2 \mathrm{~b}$ ). This shows that the charged domain wall parallel to the electrode prohibits the nucleation of new domains. Figure $8 \mathrm{~b}$ shows the case where the residual domain was switched only across the left half of the capacitor. It can be seen that the capacitor 
a

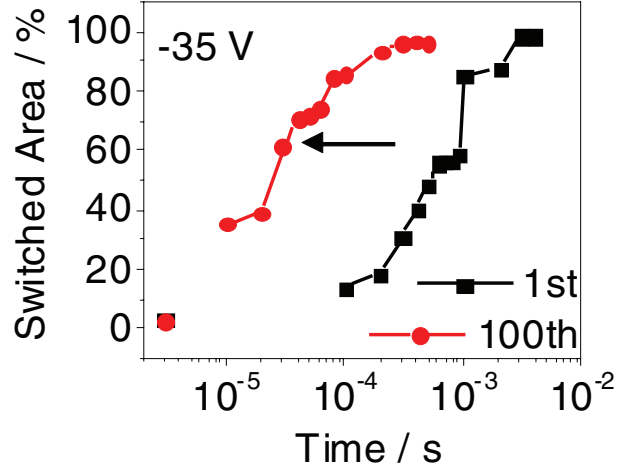

C

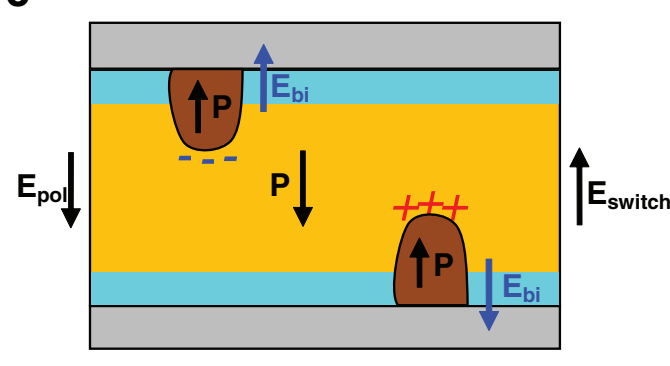

b

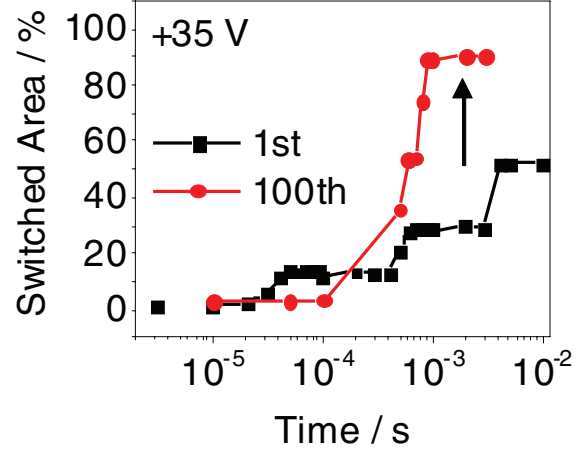

d

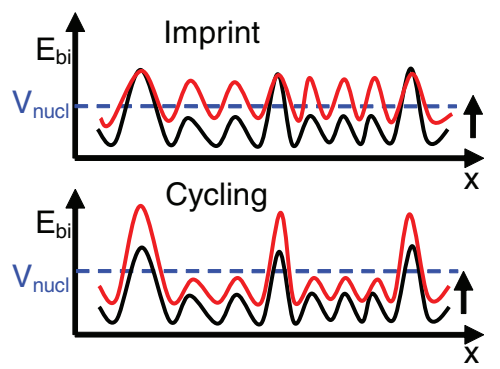

Figure 5. Main Document Only. Influence on electrical cycling on capacitor switching. Switched capacitor area vs. pulse length for the first and 100th switching cycle for switching pulses of $-35 \mathrm{~V}(\mathrm{a})$, and $35 \mathrm{~V}$ (b). c) Schematic showing the built-in field $E_{\mathrm{bi}}$ and the two possible domain nuclei with negatively and positively charged domain walls. d) Schematic drawing of variation of $E_{\mathrm{bi}}$ along the electrode/ferroelectric interface and its change with imprint and cycling.

a

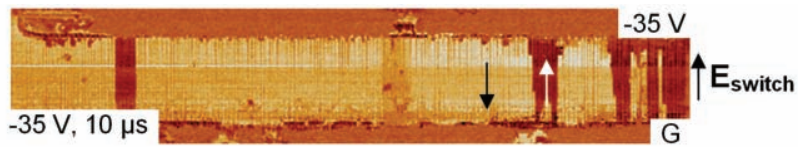

b

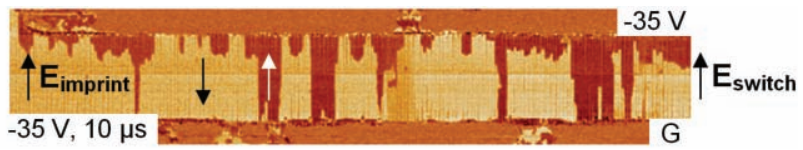

C

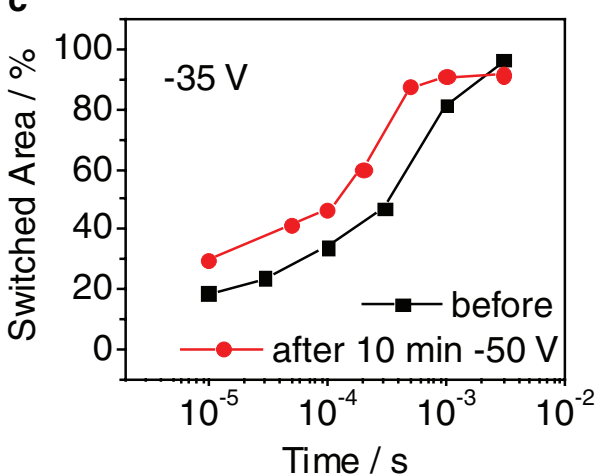

Figure 6. IP PFM images after switching with $-35 \mathrm{~V}$ and $10 \mu$ s before a) and after b) $-50 V_{\mathrm{dc}}$ imprint field was applied for $10 \mathrm{~min}$ to the capacitor. c) Influence of the dc field prior to switching on the overall switching curve. without residual domains switches normal whereas the side with the residual domains shows no complete switching after $-35 \mathrm{~V}$ and $5 \mathrm{~ms}$.

\section{Discussion}

The PFM studies of the in-plane switching of the planar capacitor structures reveal surprisingly large number of spatial symmetry breaking and memory effects that control the switching mechanisms. While some of these mechanisms (imprint, training) were previously reported and phenomenologically characterized for bulk ceramic materials, in-plane geometry allows the insight into the mesoscopic nucleation and domain wall dynamics underpinning these phenomena. These experimental observations can be summarized as follows:

i) The nucleation process is markedly asymmetric, with switching always initiated at the electrode the switching field points at, i.e., only negatively charged domain walls form.

ii) Forward and lateral domain growth is strongly affected by crystallographic structure and film morphology.

iii) The capacitor poling or switching may be incomplete, resulting in near-electrode non-switched domains that do not contribute to the nucleation and can serve as pinning centers.

These observations are discussed in detail in subsequent sections 4.1.-3. and are compared to classical ferroelectric theories. 
a
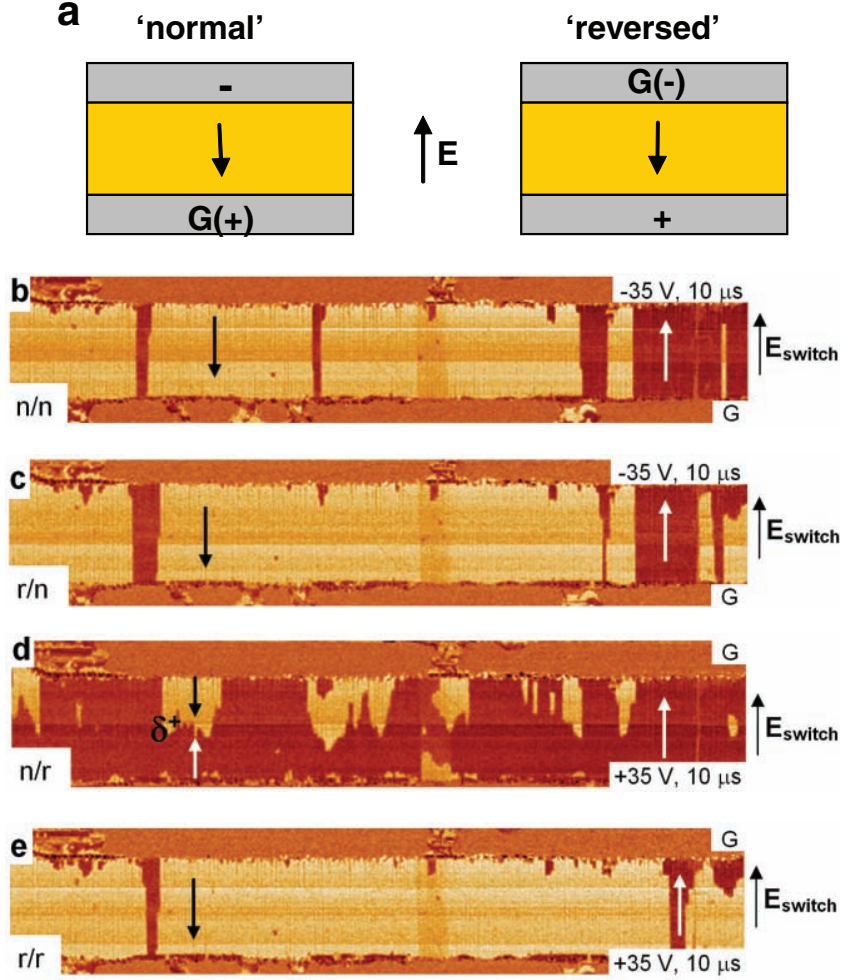

Figure 7. a) Two configurations to apply an electrical field in the same direction using the grounded electrode once with a relative positive and once with a relative negative charge. b-e) IP PFM images after poling with $|50 \mathrm{~V}|$ for $5 \mathrm{~s}$ in normal $(\mathrm{n})$ or reversed $(r)$ setup followed by switching with $|35 \mathrm{~V}|$ applied in normal or reversed setup (poling setup/switching setup).

\subsection{Domain Nucleation}

The preferential nucleation sites in one electrode in the nominally symmetric planar capacitor are compatible with the recently predicted interface stimulated scenario of switching. ${ }^{[42-44]}$ The

a

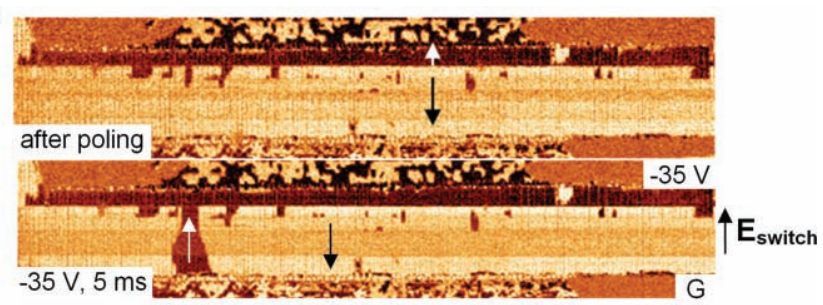

b

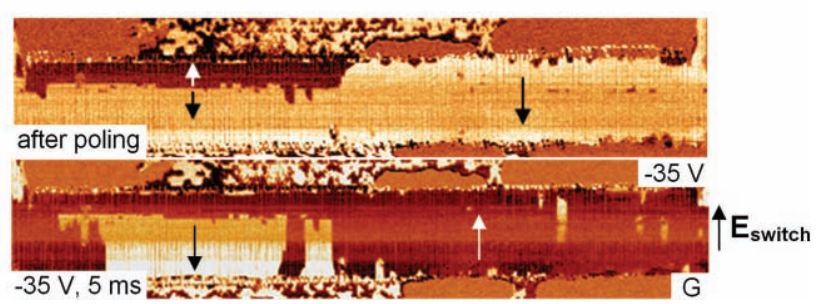

Figure 8. Capacitor switching with $-35 \mathrm{~V}$ and $5 \mathrm{~ms}$ after writing domains parallel to the top electrode across a) the full and b) half the capacitor length. ferroelectric/electrode interface is naturally a planar defect breaking the inversion symmetry. Correspondingly, the energy of a reverse domain nucleus can depend on the mutual orientation of the polarization in the nucleus and the normal to the ferroelectric/electrode interface. The driving force for this effect can be the presence of the built-in field $E_{\mathrm{bi}}$ at the interfaces, e.g. due to the work function mismatch between constitutive oxides (see Figure $5 \mathrm{c}$ ). This interface effect can be naturally rationalized by the presence of space charge layers at ferroelectricelectrode interface, e.g. due to the depletion effect of deep traps in the ferroelectric. ${ }^{[45]}$ The resulting built-in fields at the two electrodes are antiparallel to each other, thus stimulating nucleation at the electrode where the built-in field is oriented in the same direction as the switching field. ${ }^{[43]}$ In general, the higher the built-in field the lower the applied external voltage to induce domain nucleation.

Alternatively, the poling effect of the interface can be provided by a direct short-range coupling between the polarization and electrode due to chemical bonding effects. ${ }^{[46]}$ The estimates for $\mathrm{Pb}\left(\mathrm{Zr}_{x}, \mathrm{Ti}_{1-x}\right) \mathrm{O}_{3}$ showed that with the concentration of deep traps participating in the depletion of about $10^{18} \mathrm{~cm}^{-3}$, the builtin field at the interface can be as large as $50 \mathrm{kV} \mathrm{cm}^{-1}$. That means that a built-in field comparable to the typical values of the field in our experiments $\left(6 \mathrm{kV} \mathrm{cm}^{-1}\right)$ is quite realistic. The direct polarization/electrode coupling mechanism, according to the order of magnitude estimates ${ }^{[44]}$ for $\mathrm{BaTiO}_{3}$, may reduce the critical field for the reverse domain nucleation by two orders of magnitude with respect to the non-interface-assisted situation. For a given sense of switching, such reduction occurs only at one electrode.

The initiation of the capacitor switching can be characterized by the number of nucleation sites and the nucleation voltage that is determined by the average built-in field and its fluctuation along the interface due to chemical inhomogeneities, interface roughness, defects, material damage, etc. The exact distribution of built-in fields can be different for the two electrodes, which results in the different switching behavior for positive and negative switching voltages. The application of an imprint field prior to the capacitor switching strongly increases the number of nucleation sites, however, their nucleation voltage is almost unchanged (Figure 6). This suggests that imprint reduces the built-in field fluctuation without increasing the maximum builtin field, as schematically shown in Figure 5d. A possible explanation for this is the accumulation of mobile positive charge carriers at the electrode under the influence of the imprint field. On the other hand, cycling with electrical fields (i.e. repeated switching of the capacitor) lowers the nucleation voltage but the number of nucleation sites remains constant (as mentioned in 3.1.). Thus, cycling increases the average built-in field without affecting the field fluctuations, which means the built-in field is increased everywhere but the critical nucleation voltage is not exceeded in more positions than before. (Figure $5 \mathrm{~d}$ ). Cycling with electrical fields can lead to a redistribution of charge carriers that partially compensate the built-in fields, thus explaining the observed phenomena.

To complete the discussion, a complementary explanation for the preferential stabilization of the negatively charged domain walls can be given (bulk effect). The formation of the domain nucleus and the forward propagation of the domain wall 
are necessarily associated with the formation of the charged domain apex that gives rise to well-known Landauer instability. The energy of this wall will be dependent on the sign of the majority carriers in the systems, so that e.g. negatively charged domain apex will be preferentially stabilized in the system with $p$-conductivity.

It has been shown in Figure 7, that the stability of charged domain walls can be manipulated by changing the biasing conditions (Figure 7). This can be explained by the accumulation of surface screening charges that can directly affect the energy of the domain apex through the compensation of uncompensated charge. This surface screening behavior was extensively explored in the context of the transport experiments in nanotube and nanowire structures. ${ }^{[47,48]}$ In short, the accumulation of surface screening charges induced by electrode bias acts similarly to a (poorly controlled) gate electrode. By changing the bias conditions the sign of the applied switching voltage is reversed, which can introduce and accumulation negative charges of the surface that stabilize the positively charged domain walls (as opposed to normally preferred negatively charged domain walls stabilized by the p-carriers in the material), allowing switching pathways be controlled.

\subsection{Domain Growth}

The forward growth of the narrow stripe domains has been commonly observed in uniaxial ferroelectrics (ferroelectrics and non-ferroelastics), see, e.g., ${ }^{[49,50]}$ as well as multiaxial ferroelectrics. ${ }^{[51]}$ The elongated shape of the stripe domains is customarily attributed to electrostatic effects, specifically the reduction of the electrostatic energy of the bound charges at the surface of the stripe. Additionally, in the case of nucleation of the ferroelastic domains, the shape of the stripe domains corresponds to the orientation of the walls limiting the domains, which is close to that mechanically compatible. In these experiments, the orientations of the elastically compatible domain walls dividing the two polarization states involved in the switching in our experiment (see Figure 1d) can be determined as (110) and (001). ${ }^{[52]}$ Furthermore, the domain structure image $(-35 \mathrm{~V} 50 \mu \mathrm{s})$ in Figure $2 \mathrm{~b}$ is readily interpreted as the evidence for a stripe domain limited with walls of (110) orientation. For a mechanically free bulk sample this orientation is fully forbidden, since it would mechanically stress the whole sample, with the associated increase in the elastic energy of the system proportional to the volume of the sample. However, in a thin film, the mechanical strain caused by a mechanically incompatible wall will extend from the wall to a distance roughly equal to the film thickness. Correspondingly, the energy excess is proportional to the film thickness rather than to the sample size, allowing for bulk-forbidden domain configurations.

The observed sideways domain wall motion can be explained by both, sideways domain wall motion across the topography maxima or by the nucleation and forward growth of new domains. The avalanche-like capacitor switching process as well as observations of domains that do not connect both electrodes (as shown in Figure 4b,c) strongly suggest the model of the lateral domain wall motion. Since the domain walls are always correlated with topography minima, it has to be assumed that they form intrinsic pinning centers, slowing down the capacitor switching. However, after capacitor cycling the domain wall velocity was strongly increased, which improved the capacitor switching dramatically for positive and negative voltages (Figure 5).

\subsection{Role of Remnant Domains in the Switching Process}

One of the key observations during the in-plane capacitor switching is the formation of the remnant domains and their subsequent freezing. Rather than act as nucleation centers, these domains act as pinning centers for moving walls. This behavior can be rationalized in view of the present knowledge on the dynamics of charged domain walls, which act as necessary participants in the switching process. In his seminal paper, Landauer ${ }^{[53]}$ points out that screening of the polarization bound charges on a domain wall leads to a reduction of its mobility. A detailed quantitative analysis of this problem was recently performed by Mokry et al. ${ }^{[54]}$ It has been shown that the Maxwell pressure acting from the applied field to the wall is reduced with the progressive degree of screening and may even change sign. When a reverse domain grows in a film of (conductive) BFO, the wall segments bearing bound charge will be progressively screened by free carriers. This will slow the domain growth, which enables further screening, resulting in effective self-trapping of the charge wall. The domain configuration with completely screened wall segments is essentially charge-neutral and hence immobile (i.e. the ponderomotoric pressure acting on the wall in the external field is close to zero). Note that the trapping will be determined both by the switching rate and the landscape of defect-induced random fields in the vicinity of the electrodes. With the reversal of the external field, the ponderomotoric pressure changes sign, but remains small. Hence, these remnant domains do not serve as the seeds for the reverse switching.

The same is true for artificially created residual domains (Figure 8). In this case we create a charged domain wall parallel to the electrodes. After compensation of these charges the wall becomes immobile and does not take place in the capacitor switching process. This can be used to passivate parts of the capacitor as shown in Figure 8b. These observations suggest a novel strategy for fundamental studies and applications of ferroelectric devices with in-plane capacitor geometry. The passivation of regions in the capacitor can be used to localize the nucleation sites to a small number of selected locations, thus allowing to explore systematically the effect of the dissimilar structural factors on domain nucleation. From the application perspective, the soft ferroelectric lithography can be used to fabricate a number of independent active devices within a single microfabricated structure, opening pathway for further device miniaturization.

\section{Conclusions}

Polarization switching mechanisms in ferroelectric materials including nucleation and forward and lateral domain wall motion stages have been studied using model epitaxial $\mathrm{BiFeO}_{3}$ planar capacitors in a planar geometry. The choice of 
crystallographic orientation of material, electrode, and scanning direction allows single-stage switching between two [111] and [11-1] polarization direction to be explored. We demonstrate that the low-field switching is controlled by a small number of nucleation centers. Interestingly, the pre-existing domain nuclei do not act as nucleation centers, and in fact often impede switching and act as a pinning site for domain wall motion. In the pristine state of the capacitor, the switching is always initiated at the negatively charged electrode, suggesting the lower energy of the negatively charge domain walls and the presence of built-in fields at the interface. The lateral wall growth kinetics is dominated by the lateral wall pinning on surface topographic features. The interplay between the nucleation and pinning in the presence of a small number of centers leads to highly discontinuous switching curves with a large number of Barkhausen jumps. These studies allow direct visualization of the text book domain growth scenario.

Beyond observations of switching in pristine structures, we have explored the possibility to manipulate the characteristics of the capacitor switching process by using ac cycling fields, dc imprint fields, or by engineering the domain structure using the biased AFM tip. With these, all aspects of the switching process, the nucleation voltage, number of nucleation sites, domain wall stability, domain wall motion, and the actively switching capacitor volume, could be manipulated. This helps to understand the role of interface, bulk, and surface effects in the ferroelectric switching process. All these behaviors are reversible in a sense that long-term relaxation of capacitor structure results in return to original switching behavior. This opens the pathway for fundamental studies of switching processes, short-term capacitor manipulation for special applications, and miniaturization of ferroelectric devices below the lithographic limit.

Overall, the in-plane capacitors offer an ideal platform to explore domain growth processes, providing the striking illustration of the classic scenario. Furthermore, the individual steps of wall pinning and interaction with preexisting domains and structural defects can be observed, suggesting tremendous potential for future studies of single-domain dynamics.

\section{Experimental Section}

Materials: Device structures were constructed by growing an epitaxial $50 \mathrm{~nm}$ thick film of the bottom electrode material $\mathrm{SrRuO}_{3}(\mathrm{SRO})$ at $680^{\circ} \mathrm{C}$ and 100 mTorr of oxygen via pulsed laser deposition. Following growth, films were cooled to room temperature in 760 Torr of oxygen. The in-plane electrode structures were achieved through a photolithography step to define the device structures. The electrode patterns used in this study were controlled to have a separation of $6 \mu \mathrm{m}$ and $50 \mu \mathrm{m}$ length. Removal of the surrounding SRO materials was achieved via an ion milling step to etch away the surrounding SRO material down to the STO substrate. The photoresist was then removed from the samples and $80 \mathrm{~nm}$ thick BFO films were grown via pulsed laser deposition at $700 \mathrm{C}$ in 100 mTorr of oxygen before being cooled to room temperature in 760 Torr of oxygen.

Experimental Set-Up: Piezoresponse force microscopy (PFM) was done using a commercial system (Veeco, Dimensions). The tip was oriented along the [110] direction to distinguish between the two domain variants through the lateral PFM signal. For domain imaging, the ac voltage of $1.5 \mathrm{~V}$ at $30 \mathrm{kHz}$ is applied directly to the scanning PFM tip.
For capacitor switching, the tip is retracted and square voltage pulses of different lengths and amplitudes are applied to the planar $\mathrm{SrRuO}_{3}$ electrodes. The minimum pulse length is limited by the voltage amplifier raise time and was around $10 \mu \mathrm{s}$. The minimum positive pulse voltage that switched the whole capacitor was found to be $35 \mathrm{~V}$ across the $6 \mu \mathrm{m}$ electrode gap, corresponding to a switching field of $\sim 60 \mathrm{kV} \mathrm{cm}^{-1}$. All switching experiments were conducted at this field since higher fields did not allow observing the initial stages of the switching process within the pulse length limit.

\section{Acknowledgements}

Research sponsored by the Division of Scientific User Facilities, Office of Basic Energy Sciences, U.S. Department of Energy (SVK). N.B. acknowledges Alexander von Humboldt foundation. Y.H.C. would also like to acknowledge the support of the National Science Council, R.O.C., under contract NO. NSC-98-2119-M-009-016. The work at Berkeley is supported by the Director, Office of Science, Office of Basic Energy Sciences, Materials Sciences Division of the U.S. Department of Energy under contract No. DE-ACO2-05CH1123. Correspondence should be addressed to balken@ornl.gov

Received: March 12, 2010

Revised: May 20, 2010

Published online: August 17, 2010

[1] J. F. Scott, Ferroelectric memories, Springer, Heidelberg, Germany 2000.

[2] a. L. Esaki, R. B. Laibowitz, P. J. Stiles, IBM Tech. Disc. Bull. 1971, 13, 2161; b) L. L. Chang, L. Esaki, IBM Tech. Disc. Bull. 1971, 14, 1250.

[3] I. M. Ross, U. S. Patent 1957, Nr. 2, 791, 760.

[4] R. Wolf, P. W. M. Blom, M. P. C. Krijn, U. S. Patent 1996, Nr. 5, 541, 422.

[5] E. Y. Tsymbal, H. Kohlstedt, Science 2006, 313, 181.

[6] P. Maksymovych, S. Jesse, P. Yu, R. Ramesh, A. P. Baddorf, S. V. Kalinin, Science 2009, 324, 1421.

[7] V. Garcia, S. Fusil, K. Bouzehouane, S. Enouz-Vedrenne, N. D. Mathur, A. Barthelemy, M. Bibes, Nature 2009, 460, 81.

[8] A. Gruverman, D. Wu, H. Lu, Y. Wang, H. W. Jang, C. M. Folkman, M. Ye. Zhuravlev, D. Felker, M. Rzchowski, C.-B. Eom, E. Y. Tsymbal, Nano Lett. 2009, 9, 3539.

[9] M. Alexe, C. Harnagea, D. Hesse, U. Gösele, Appl. Phys. Lett. 1999, 75, 1793

[10] W. Lee, H. Han, A. Lotnyk, M. A. Schubert, S. Senz, M. Alexe, D. Hesse, S. Baik, U. Gösele, Nat. Nanotechnol. 2008, 3, 402.

[11] S. Shur, E. Rumyantsev, S. Makarov, J. Appl. Phys. 1998, 84, 445.

[12] T. K. Song, S. Aggarwal, Y. Gallais, B. Nagaraj, R. Ramesh, Appl. Phys. Lett. 1998, 73, 3366.

[13] O. Lohse, M. Grossmann, U. Boettger, D. Bolten, J. Appl. Phys. 2001, 89, 2332.

[14] A. K. Tagantsev, I. Stolichnov, N. Setter, Phys. Rev. B 2002, 66, 214109.

[15] S. Prasertchoung, V. Nagarajan, Z. Ma, R. Ramesh, Appl. Phys. Lett. 2004, 84, 3130.

[16] J. Y. Jo, D. J. Kim, Y. S. Kim, S. B. Choe, T. K. Song, J. G. Yoon, T. W. Noh, Phys. Rev. Lett. 2006, 97, 247602.

[17] A. Gruverman, Appl. Phys. Lett. 1999, 75, 1452.

[18] S. Hong, E. L. Colla, E. Kim, D. V. Taylor, A. K. Tagantsev, P. Muralt, K. No, N. Setter, J. Appl. Phys. 1999, 86, 607.

[19] H. Fujisawa, T. Yagi, M. Shimizu, H. Niu, Ferroelectrics 2002, 269, 21.

[20] A. Gruverman, B. J. Rodriguez, C. Dehoff, J. D. Waldrep, A. I. Kingon, R. J. Nemanich, Appl. Phys Lett. 2005, 87, 082902. 
[21] D. J. Kim, J. Y. Jo, T. H. Kim, S. M. Yang, B. Chen, Y. S. Kim, T. W. Noh, Appl. Phys. Lett. 2007, 91, 132903.

[22] A. Gruverman, D. Wu, H. J. Fan, I. Vrejoiu, M. Alexe, R. J. Harrison, J. F. Scott, J. Phys.: Condens. Matter 2008, 20, 342201.

[23] A. Gruverman, D. Wu, J. F. Scott, Phys. Rev. Lett. 2008, 100, 097601.

[24] P. Bintachitt, S. Trolier-McKinstry, K. Seal, S. Jesse, S. V. Kalinin, Appl. Phys. Lett. 2009, 94, 042906.

[25] S. Hong, B. Ecabart, E. L. Colla, N. Setter, Appl. Phys. Lett. 2004, 84 2382.

[26] I. Stolichnov, L. Malin, E. Colla, A. K. Tagantsev, N. Setter, Appl. Phys. Lett. 2005, 86, 012902.

[27] X. M. Lu, F. Schlaphof, S. Grafstrom, C. Loppacher, L. M. Eng, G. Suchaneck, G. Gerlach, Appl. Phys. Lett. 2002, 81, 3215.

[28] J. S. Liu, H. Z. Zeng, A. L. Kholkin, J. Phys. D: Appl. Phys. 2007, 40, 7053.

[29] R. Gysel, A. K. Tagantsev, I. Stolichnov, N. Setter, Appl. Phys. Lett. 2006, 89, 082906

[30] P. Shafer, F. Zavaliche, Y. H. Chu, P. L. Yang, M. P. Cruz, R. Ramesh, Appl. Phys. Lett. 2007, 90, 202909.

[31] Y. H. Chu, L. W. Martin, M. B. Holcomb, M. Gajek, S. J. Han, Q. He, N. Balke, C. H. Yang, D. Lee, W. Hu, Q. Zhan, P. L. Yang, A. Fraile-Rodríguez, A. Scholl, S. X. Wang, R. Ramesh, Nat. Mater. 2008, 7, 478.

[32] S. K. Streiffer, C. B. Parker, A. E. Romanov, M. J. Lefevre, L. Zhao, J. S. Speck, W. Pompe, C. M. Foster, G. R. Bai, J. Appl. Phys. 1998, 83, 2742.

[33] Y. H. Chu, M. P. Cruz, C. H. Yang, L. W. Martin, P. L. Yang, J. X. Zhang, K. Lee, P. Yu, L. Q. Chen, R. Ramesh, Adv. Mater. 2007, 19, 2662.

[34] H. Zheng, F. Straub, Q. Zhan, P.-L. Yang, W.-K. Hsieh, F. Zavaliche, Y.-H. Chu, U. Dahmen, R. Ramesh, Adv. Mater. 2006, 18, 2747.

[35] N. Bassiri-Gharb, I. Fujii, E. Hong, S. Trolier-McKinstry, D. V. Taylor, D. Damjanovic, J. Electroceram. 2007, 19, 47.
[36] S. Buhlmann, P. Muralt, Adv. Mater. 2008, 20, 3090.

[37] G. W. Pabst, L. W. Martin, Y.-H. Chu, R. Ramesh, Appl. Phys. Lett. 2007, 90, 072902

[38] K. Abe, N. Yanase, T. Yasumoto, T. Kawakubo, J. Appl. Phys. 2002 $91,323$.

[39] W. L. Warren, D. Dimos, G. E. Pike, B. A. Tuttle, M. V. Raymond, R. Ramesh, J. T. Evans, Appl. Phys. Lett. 1995, 67, 866.

[40] M. Grossmann, O. Lohse, D. Bolten, U. Boettger, T. Schneller R. Waser, J. Appl. Phys. 2002, 92, 2680.

[41] A. K. Tagantsev, I. Stolichnov, N. Setter, J. S. Cross, J. Appl. Phys. 2004, 96, 6616

[42] V. Janovec, Czech. J. Phys. 1959, 9, 468.

[43] A. K. Tagantsev, C. Pawlaczyk, K. Brooks, N. Setter, Integr. Ferroelectr. 1994, $4,1$.

[44] G. Gerra, A. K. Tagantsev, N. Setter, Phys. Rev. Lett. 2005, 94, 107602.

[45] C. J. Brennan, Integr. Ferroelectr. 1995, 7, 93.

[46] G. Gerra, A. K. Tagantsev, N. Setter, Phys. Rev. Lett. 2007, 98, 207601.

[47] S. V. Kalinin, D. A. Bonnell, T. Alvarez, X. Lei, Z. Hu, J. H. Ferris, Q. Zhang, S. Dunn, Nano Lett. 2002, 2, 589.

[48] S. V. Kalinin, J. Shin, S. Jesse, D. Geohegan, A. P. Baddorf, Y. Lilach, M. Moskovits, A. Kolmakov, J. Appl. Phys. 2005, 98, 044503.

[49] A. Kuroda, S. Kurimura, Y. Uesu, Appl. Phys. Lett. 1996, 69, 1565.

[50] J. Bornarel, J. Lajzerow, Ferroelectrics 1972, 4, 177.

[51] J. Kobayashi, Phys. Status Solidi 1967, 21, 151.

[52] S. K. Streiffer, C. B. Parker, A. E. Romanov, M. J. Lefevre, L. Zhao, J. S. Speck, W. Pompe, C. M. Foster, G. R. Bai, J. Appl. Phys. 1998, 83, 2742.

[53] R. Landauer, J. Appl. Phys. 1957, 28, 227.

[54] P. Mokry, A. K. Tagantsev, J. Fousek, Phys. Rev. B 2007, 75, 094110. 